\title{
Three histologically distinct cancers of the uterine corpus: A case report and review of the literature
}

\author{
HISASHI MASUYAMA ${ }^{1}$, JUNKO HARAGA ${ }^{1}$, TAKASHI NISHIDA ${ }^{1}$, CHIKAKO OGAWA $^{1}$, \\ TOMOYUKI KUSUMOTO $^{1}$, KEIICHIRO NAKAMURA ${ }^{1}$, NORIKO SEKI ${ }^{1}$, \\ HIROYUKI YANAI $^{2}$ and YUJI HIRAMATSU ${ }^{1}$
}

\author{
${ }^{1}$ Department of Obstetrics and Gynecology, Okayama University Graduate School of Medicine, Dentistry \\ and Pharmaceutical Sciences; ${ }^{2}$ Department of Pathology, Okayama University Hospital, Okayama 700-8558, Japan
}

Received October 2, 2015; Accepted January 29, 2016

DOI: $10.3892 / \operatorname{mco} .2016 .770$

\begin{abstract}
Collision tumors, which are characterized by the coexistence of two or more completely distinct and independent tumors in the uterine corpus, are very rare. A collision tumor is mainly composed of two distinct tumor types, epithelial and mesenchymal. To the best of our knowledge, there has only been a single case in which a choriocarcinoma with an endometrial carcinoma were coexistent but histologically distinct. We herein report the first case of a collision tumor in a 52-year-old woman, with a history of two pregnancies and two deliveries. The collision tumor was composed of three histologically distinct neoplasms in the uterine corpus, namely an endometrioid carcinoma, an undifferentiated carcinoma and a choriocarcinoma. The patient underwent hysterectomy, bilateral adnexectomy and pelvic lymph node dissection, followed by six cycles of adjuvant chemotherapy with paclitaxel/carboplatin due to the high risk of endometrial cancer, and an additional five cycles of chemotherapy with methotrexate, as the $\beta$-human chorionic gonadotropin level was beyond the normal range. Following adjuvant chemotherapy, the tumor markers were within normal limits and no relapses of the cancer have been observed during 1 year of follow-up. Diagnosing a collision tumor prior to surgery is difficult if the neoplasms are in close proximity, or if one of the tumors predominates. Careful pathological examination is crucial for accurately diagnosing the neoplasms in a collision tumor and ensuring appropriate management and a favorable prognosis.
\end{abstract}

Correspondence to: Dr Hisashi Masuyama, Department of Obstetrics and Gynecology, Okayama University Graduate School of Medicine, Dentistry and Pharmaceutical Sciences, 2-5-1 Shikata, Kita-ku, Okayama 700-8558, Japan

E-mail:masuyama@cc.okayama-u.ac.jp

Key words: collision tumor, endometrioid adenocarcinoma, choriocarcinoma, undifferentiated carcinoma, uterine corpus

\section{Introduction}

Tumors that occur in the uterine corpus include epithelial, mesenchymal, mixed epithelial and mesenchymal, miscellaneous, lymphoid and myeloid and secondary tumors, as well as trophoblastic disease (1). Endometrial cancer is the third most common cause of death among gynecological cancers, following ovarian and cervical cancer, with increasing incidence rates in several countries (2). This may be attributed to the increasing number of elderly individuals and increasing rates of obesity (2). Endometrial cancer includes several carcinomas, which are divided into type I and II cancers and include endometrioid adenocarcinomas, mucinous adenocarcinomas, serous carcinomas, clear-cell carcinomas and several other rare forms, such as neuroendocrine tumors, including small-cell, mixed, undifferentiated and dedifferentiated carcinomas (3).

In addition, choriocarcinoma is a malignant trophoblastic tumor, usually of the placenta, that may also occur in the uterine corpus. Choriocarcinoma belongs to the malignant end of the spectrum of gestational trophoblastic disease, with $20 \%$ of this disease occurring after normal pregnancy (4). However, it is rare for trophoblastic disease to present in the uterus of postmenopausal women (5). Furthermore, non-gestational choriocarcinomas occur in the absence of a preceding gestation, and often tend to occur as a component of an ovarian germ cell tumor (6). Almost all primary non-gestational choriocarcinomas of the female genital tract have been described in the ovaries $(7,8)$.

The number of reports in the literature regarding endometrial cancer in which choriocarcinoma differentiation occurs in the uterine corpus is limited (9), with only a single report of coexistence of a mixed adenocarcinoma and a choriocarcinoma (10). We herein report the first case of a collision tumor in the uterine corpus composed of three histologically distinct cancers, namely an endometrioid carcinoma, an undifferentiated carcinoma and a choriocarcinoma.

\section{Case report}

Our patient was a 52-year-old woman with a history of two pregnancies and two deliveries; the last delivery was when she 

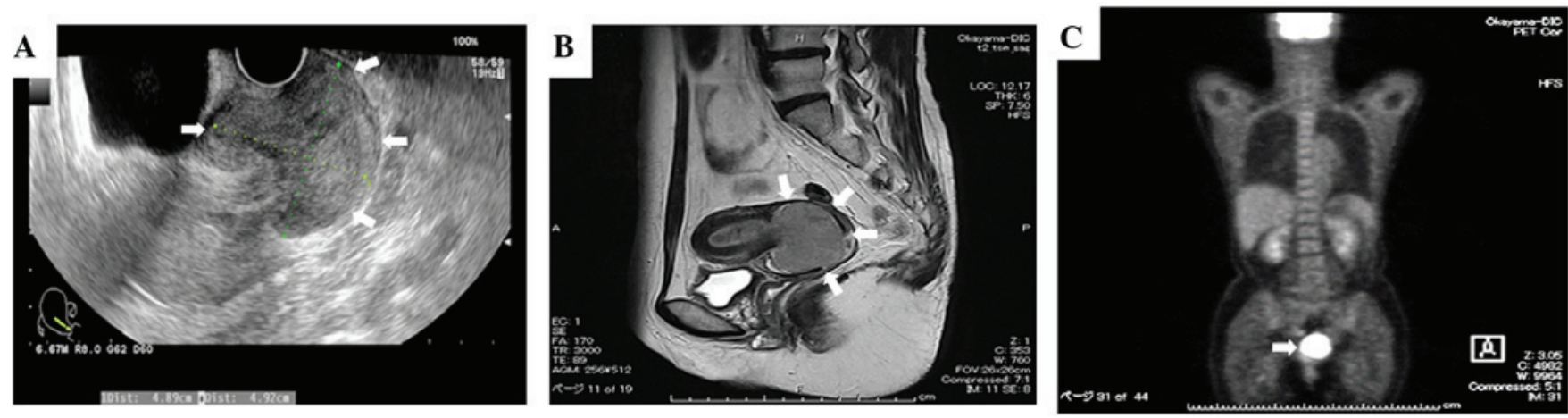

Figure 1. (A) Transvaginal ultrasonography revealed a mixed echogenic mass in the cervix. (B) Magnetic resonance imaging showed a hyperintense cervical mass invading the lower uterine segment. (C) Positron emission tomography-computed tomography revealed intense fluorodeoxyglucose activity correlating with the cervical and endometrial masses, without signs of metastasis to other organs or to the pelvic and para-aortic lymph nodes.

was 28 years old. The patient was $156 \mathrm{~cm}$ in height, weighed $51 \mathrm{~kg}$, and had a body mass index of $20.95 \mathrm{~kg} / \mathrm{m}^{2}$. The history of menstrual cycles was regular and she had gone through menopause at the age of 51 years. The surgical history included an appendectomy at the age of 19 years, but there was no family history of cancer. The patient consulted a gynecologist with a main complaint of moderate genital bleeding. A 35x30-mm mass lesion, which bled easily, was detected in the cervix.

The patient was referred to our hospital and, after undergoing a detailed examination, received further treatment due to the suspicion of cervical cancer. During the first visit to our hospital, a pelvic examination revealed an enlarged cervix, while the digital examination revealed extension to the parametrium on either side of the uterus. The colposcopic examination revealed that the invasive carcinoma was a large polypoid mass in the cervical canal. The cytological diagnosis of the cervical lesion was a high-grade squamous intraepithelial lesion and the pathological diagnosis, using a punch biopsy, indicated a squamous cell carcinoma (SCC). Transvaginal ultrasonography revealed a mixed echogenic mass in the cervix, sized 49x49x57 mm (Fig. 1A). Magnetic resonance imaging (MRI) revealed a hyperintense cervical mass sized $55 \times 51 \times 52 \mathrm{~mm}$, invading the lower uterine segment (Fig. 1B). Positron emission tomography-computed tomography (CT) revealed intense fludeoxyglucose activity correlating with a cervical and endometrial mass, without signs of metastasis to other organs or to the pelvic and paraaortic lymph nodes (Fig. 1C). The tumor marker carbohydrate antigen (CA)-125 was elevated (91.8 U/ml), but other markers, including carcinoembryonic antigen, CA19-9 and SCC antigen, were within the normal range.

With an initial diagnosis of stage IB2 cervical cancer, the patient underwent radical hysterectomy, bilateral adnexectomy and pelvic lymph node dissection. The abdominal cavity was free of ascites, disseminated lesions and adhesions to adjacent organs. Three distinct tumors were identified in the resected specimen, one in the lower segment, one in the middle segment and one in the fundus of the uterine corpus (Fig. 2A). The histopathological examination of the masses revealed that they were three histologically distinct cancers: An undifferentiated carcinoma of the lower segment, invading half the thickness of the myometrium (Fig. 2B); an endometrioid adenocarcinoma with squamous differentiation, grade 2 , confined to the endometrium in the middle segment (Fig. 2C); and a choriocarcinoma with lymphovascular space invasion, but without an endometrioid adenocarcinoma component, in the fundus of the uterine corpus (Fig. 2D), metastasizing to the lymph nodes of the left external iliac artery. There was no communication between the three lesions in the uterine corpus.

The clinical diagnosis was stage IIIC1 endometrial cancer [International Federation of Gynecology and Obstetrics 2008 (1)] and choriocarcinoma. The patient was diagnosed with three histologically distinct neoplasms in the uterine corpus. After the operation, chemotherapy with paclitaxel $175 \mathrm{mg} / \mathrm{m}^{2}$ plus carboplatin area under the curve $=6$ ( TC regimen) was administered, due to the high risk of endometrial cancer. Six treatment cycles were completed without dose reduction or suspension of treatment. The $\beta$-human chorionic gonadotropin (hCG) level was $19.5 \mathrm{mIU} / \mathrm{ml}$ when the pathological results of the coexistence with a choriocarcinoma were obtained, and the $\beta$-hCG level $(1.0 \mathrm{mIU} / \mathrm{ml})$ after six cycles of TC was above the normal range $(0-0.5 \mathrm{mIU} / \mathrm{ml})$, although other tumor markers were within normal limits, and CT revealed no cancer recurrence or metastasis. Five additional cycles using methotrexate (20 $\mathrm{mg} / \mathrm{m}^{2}$ body surface x 5 days) were administered and completed without dose reduction or suspension of treatment. After the five cycles, the tumor markers, including the $\beta$-hCG level, were within normal limits, without cancer relapse during 1 year of follow-up.

\section{Discussion}

We reported a case with three histologically distinct cancers in the uterine corpus: An endometrioid carcinoma, an undifferentiated carcinoma and a choriocarcinoma. There are several reports in the literature on endometrial cancer with choriocarcinoma differentiation, with two distinct components in the primary tumor, and the prognosis of this tumor type is reported to be poor (9). However, only a single case of a histologically distinct coexistence of a mixed adenocarcinoma and a choriocarcinoma in the uterine corpus has been reported to date (10).

Collision tumors are an uncommon phenomenon characterized by the coexistence of two completely distinct and independent tumors at the same site. The two morphologically separate tumors are sharply demarcated from each other. This 
Table I. Summary of histological diagnosis of collision tumors of the uterine corpus reported in the literature.

\begin{tabular}{lc}
\hline Histological diagnosis & No. \\
\hline Two distinct tumors & $($ Refs.) \\
Endometrioid carcinoma/serous carcinoma/leiomyosarcoma or endometrial stromal sarcoma & 7 \\
Endometrioid carcinoma/carcinosarcoma & 1 \\
Serous carcinoma/small cell carcinoma & 1 \\
Mixed cell carcinoma/choriocarcinoma & 1 \\
Three distinct tumors & $(13-17)$ \\
Serous carcinoma/endometrioid carcinoma/carcinosarcoma & $(19)$ \\
Undifferentiated carcinoma/endometrioid carcinoma/choriocarcinoma & 1 \\
Total & 1 \\
\hline
\end{tabular}
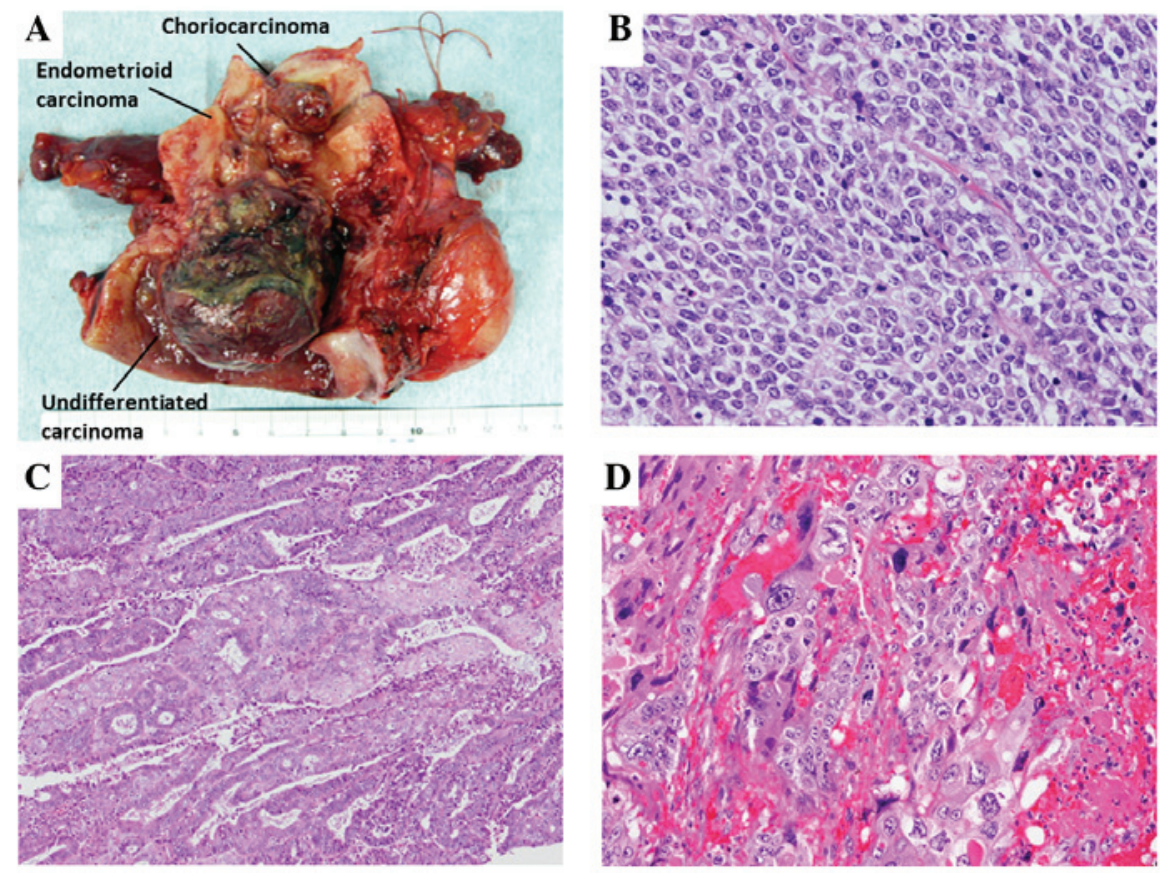

Figure 2. (A) Surgical specimen displaying three distinct lesions in the uterine corpus. Histopathological examination of the hysterectomy specimen revealed: (B) An undifferentiated carcinoma invading half the thickness of the myometrium in the lower segment; (C) an endometrioid adenocarcinoma with squamous differentiation, grade 2, confined to the endometrium in the middle segment; and (D) a choriocarcinoma with lymphovascular space invasion in the fundus. Hematoxylin and eosin staining; magnification, x200.

entity is distinct from neoplasms demonstrating heterologous or mixed elements (11). Collision tumors have been reported in various organs, such as the ovary and uterus, as well as in the esophagus, stomach, colon, lung, skin, thyroid gland and breast $(12,13)$.

In patients with collision tumors of the uterine corpus, the median age is 64.9 years (range, $47-85$ years); the histological diagnoses in previously reported collision tumors of the uterine corpus are summarized in Table I (10,13-20). Several reports determined that collision tumors of the uterine corpus were mainly composed of two distinct tumors, an endometrial carcinoma (endometrioid or serous adenocarcinoma) and a mesenchymal tumor (leiomyosarcoma or endometrial stromal sarcoma). However, in a recent report, three distinct endometrial cancers were found in the uterine corpus, a malignant mixed Müllerian tumor, a serous adenocarcinoma and an endometrioid adenocarcinoma (20). In the present case report, we identified three histologically distinct lesions in the uterine corpus, including an endometrioid carcinoma, an undifferentiated carcinoma and a choriocarcinoma.

Choriocarcinomas of the uterine corpus are rare, particularly after menopause (21). Choriocarcinomas of gestational and non-gestational origin have a distinct prognosis, but cannot be distinguished by routine histological examination (22). Molecular genetic analysis may reliably identify the genetic origin of a pure ovarian choriocarcinoma (23-25); however, as this is an unestablished and costly technique with limited availability, it was not used it in this case. However, our patient was in the high-risk group of endometrial cancer due to the lymph node metastasis and the histological type, i.e., undifferentiated carcinoma (26). Thus, the patient received six cycles of TC as adjuvant chemotherapy for 
high-risk endometrial cancer and an additional five cycles of chemotherapy with MTX, as the $\beta$-hCG level after six cycles of $\mathrm{TC}$ remained above the normal range.

Collision tumors of the uterine corpus are rare, with only 10 cases of two distinct tumors and 1 case of three distinct tumors reported thus far. Moreover, there was only 1 case of a collision tumor composed of a choriocarcinoma with a histologically distinct endometrial carcinoma. To the best of our knowledge, this is the first report of a collision tumor composed of three histologically distinct neoplasms, including a choriocarcinoma, an endometrioid adenocarcinoma and an undifferentiated carcinoma. The diagnosis of a collision tumor prior to surgery is difficult if the neoplasms are located in close proximity, or one tumor predominates compared with the others, as in the present case. Careful pathological examination is crucial for accurately diagnosing the neoplasms constituting the collision tumor in order to prescribe the necessary treatment for a favorable prognosis.

\section{References}

1. Kurman RJ, Carcangiu ML, Herrington CS and Young RH (eds): WHO classification of tumours of female reproductive organs In: WHO Classification of Tumours. Vol 6. 4th edition. IARC Press, Lyon, pp122-167, 2014.

2. Stewart BW and Wild CP (eds): World Cancer Report 2014. Chapter 5.12. IARC Press, Lyon, 2014.

3. Hoffman BL, Schorge JO, Schaffer JI, Halvorson LM, Bradshaw KD and Cunningham FG: Endometrial cancer. In: Williams Gynecology. Hoffman BL, Schorge JO, Schaffer JI, Halvorson LM, Bradshaw KD and Cunningham FG (eds). 2nd edition. McGraw-Hill, New York, pp817-838, 2012.

4. Lurain JR: Gestational trophoblastic disease II: Classification and management of gestational trophoblastic neoplasia. Am J Obstet Gynecol 204: 11-18, 2011.

5. Chittenden B, Ahamed E and Maheshwari A: Choriocarcinoma in a postmenopausal woman. Obstet Gynecol 114: 462-465, 2009.

6. Russell P and Farnsworth A: Non-gestational choriocarcinomas. In: Surgical Pathology of the Ovaries. Russell P and Farnsworth A (eds.). 2nd edition. Churchill Livingstone, Edinburgh, pp263-264, 1997.

7. Jiao LZ, Xiang Y, Feng FZ, Wan XR, Zhao J, Cui QC and Yang XY: Clinical analysis of 21 cases of nongestational ovarian choriocarcinoma. Int J Gynecol Cancer 20: 299-302, 2010.

8. Haruma T, Ogawa C, Nishida T, Kusumoto T, Nakamura K, Seki N, Katayama T and Hiramatsu Y: Pure choriocarcinoma of the ovary in Silver-Russell syndrome. Acta Med Okayama 69: $183-188,2015$.

9. Ishida $\mathrm{M}$ and Okabe $\mathrm{H}$ : Endometrioid adenocarcinoma with choriocarcinomatous differentiation: A case report and review of the literature. Oncol Lett 6: 655-658, 2013.

10. Gao HJ, Zhou W, Zhang XF, Zhou CY and Qian JH: Coexistent gestational choriocarcinoma and mixed adenocarcinoma of the uterus. Eur J Gynaecol Oncol 34: 362-367, 2013.
11. Van Eeden S, Nederlof PM, Taal BG, Offerhaus GJ and Van Velthuysen ML: A tumour with a neuroendocrine and papillary serous component: Two or a pair? J Clin Pathol 55: 710-714, 2002.

12. Singh AK and Singh M: Collision tumours of ovary: A very rare case series. J Clin Diagn Res 8: FD14-FD16, 2014.

13. Patwardhan JR and Gadgil RK: Collision tumour of the uterus. Indian J Cancer 6: 194-197, 1969.

14. Lifschitz-Mercer B, Czernobilsky B, Dgani R, Dallenbach-Hellweg G, Moll $R$ and Franke WW: Immunocytochemical study of an endometrial diffuse clear cell stromal sarcoma and other endometrial stromal sarcomas. Cancer 59: 1494-1499, 1987.

15. Sreenan JJ and Hart WR: Carcinosarcomas of the female genital tract. A pathologic study of 29 metastatic tumors: Further evidence for the dominant role of the epithelial component and the conversion theory of histogenesis. Am J Surg Pathol 19: 666-674, 1995.

16. Lam KY, Khoo US and Cheung A: Collision of endometrioid carcinoma and stromal sarcoma of the uterus: A report of two cases. Int J Gynecol Pathol 18: 77-81, 1999.

17. Gaertner EM, Farley JH, Taylor RR and Silver SA: Collision of uterine rhabdoid tumor and endometrioid adenocarcinoma: A case report and review of the literature. Int J Gynecol Pathol 18: 396-401, 1999.

18. Takahashi Y and Inoue T: Hepatoid carcinoma of the uterus that collided with carcinosarcoma. Pathol Int 53: 323-326, 2003.

19. Shaco-Levy R, Manor E, Piura B and Ariel I: An unusual composite endometrial tumor combining papillary serous carcinoma and small cell carcinoma. Am J Surg Pathol 28: 1103-1106, 2004

20. Jang KS, Lee WM, Kim YJ and Cho SH: Collision of three histologically distinct endometrial cancers of the uterus. J Korean Med Sci 27: 89-92, 2012.

21. Zhao J, Xiang Y, Wan XR, Feng FZ, Cui QC and Yang XY: Molecular genetic analyses of choriocarcinoma. Placenta 30: 816-820, 2009.

22. Wang Y, Yang Y, Teng F, Zhang H and Xue F: Pure nongestational uterine choriocarcinoma in a postmenopausal Chinese woman confirmed with short tandem repeat analysis. Am J Obstet Gynecol 211: e1-e3, 2014.

23. Hirata Y, Yanaihara N, Yanagida S, Fukui K, Iwadate K, Kiyokawa T and Tanaka T: Molecular genetic analysis of nongestational choriocarcinoma in a postmenopausal woman: A case report and literature review. Int J Gynecol Pathol 31: 364-368, 2012.

24. Fisher RA, Newlands ES, Jeffreys AJ, Boxer GM, Begent RH, Rustin GJ and Bagshawe KD: Gestational and nongestational trophoblastic tumors distinguished by DNA analysis. Cancer 69: 839-845, 1992.

25. Fisher RA, Savage PM, MacDermott C, Hook J, Sebire NJ, Lindsay I and Seckl MJ: The impact of molecular genetic diagnosis on the management of women with hCG-producing malignancies. Gynecol Oncol 107: 413-419, 2007.

26. Johnson N, Bryant A, Miles T, Hogberg T and Cornes P: Adjuvant chemotherapy for endometrial cancer after hysterectomy. Cochrane Database Syst Rev: CD003175, 2011. 